\title{
Large-scale periodicity in the distribution of QSO absorption-line systems
}

\author{
A. I. Ryabinkov and A. D. Kaminker
}

\begin{abstract}
The spatial-temporal distribution of absorption-line systems (ALSs) observed in QSO spectra within the cosmological redshift interval $z=0.0-4.3$ is investigated on the base of our updated catalog of absorption systems. We consider so called metallic systems including basically lines of heavy elements. The sample of the data displays regular variations (with amplitudes $\sim 15-20 \%$ ) in the $z$-distribution of ALSs as well as in the $\eta$-distribution, where $\eta$ is a dimensionless line-of-sight comoving distance, relatively to smoother dependences. The $\eta$-distribution reveals the periodicity with period $\Delta \eta=0.036 \pm 0.002$, which corresponds to a spatial characteristic scale $(108 \pm 6) h^{-1} \mathrm{Mpc}$ or (alternatively) a temporal interval $(350 \pm 20) h^{-1} \mathrm{Myr}$ for the $\Lambda \mathrm{CDM}$ cosmological model. We discuss a possibility of a spatial interpretation of the results treating the pattern obtained as a trace of an order imprinted on the galaxy clustering in the early Universe.
\end{abstract}

Keywords galaxies: quasars: absorption lines - galaxies: high-redshifts

\section{Introduction}

In our previous papers (e.g., Kaminker et al. 2000, Ryabinkov et al. 2007, hereafter Paper I) we studied the spatial-temporal distribution of absorption-line systems (ALSs) imprinted in spectra of quasars (QSOs). We regard that the redshifts $z_{a}$ of absorption lines are cosmological, and detected ALSs are associated with ionized gas in intervening galaxies or clusters of galaxies at cosmological distances along lines of sight to original QSOs. Thus a representative sample of ALSs is

A. I. Ryabinkov and A. D. Kaminker

Ioffe Physical-Technical Institute, Politekhnicheskaya 26, 194021 St. Petersburg, Russia considered to trace the distribution of matter between the observer and QSOs, as well as physical processes occurred in different epochs of the cosmological evolution.

In Paper I we explored 2003 ALSs in the redshift range $z=0.0-3.7$ from our catalog of absorption systems (Ryabinkov et al. 2003) and restricted ourselves by consideration of so called metallic systems including lines of heavy elements. ALSs registered in a spectrum of a quasar might comprise up to 20 - 30 absorption lines predominantly within an interval $\sim 3000-$ $8000 \AA$. It was shown that the $z$-distribution of ALSs displays a pattern of alternating maxima (peaks) and minima (dips) which are statistically significant against a smoother dependence (trend). The positions of the peaks and dips obtained for different hemispheres on the sky turned out to be independent (within statistical uncertainties) of observation directions. Moreover, it was shown that the sequence of peaks and dips reveals a certain regularity. The power spectrum calculated with using a rescaling function $\tau(z)$ (Eqs. (11) and (12) of Paper I) displays the peak at the significance level exceeding $4 \sigma$ relatively to the hypothesis of the uniform distribution of ALSs along the axis $\tau(z)$.

In this paper we present the results of slightly extended statistical analysis performed by similar methods as we used in Paper I but in a new bearing. The principle point of this paper is the analysis of the ALSs distribution in the comoving coordinate system (CS). Such a representation enables us to find the periodicity of the distribution and treat it as a trace of partly ordered spatial structures.

The upper panel of Fig. 1 demonstrates three $z$ distributions $N_{\mathrm{AS}}(z)$ of ALSs containing absorption lines of heavy elements within the redshift interval $z=0.0-4.3$. One of them is obtained using 847 systems from the catalog by Junkkarinen et al. (1991), the second one is based on 2003 systems registered in the 

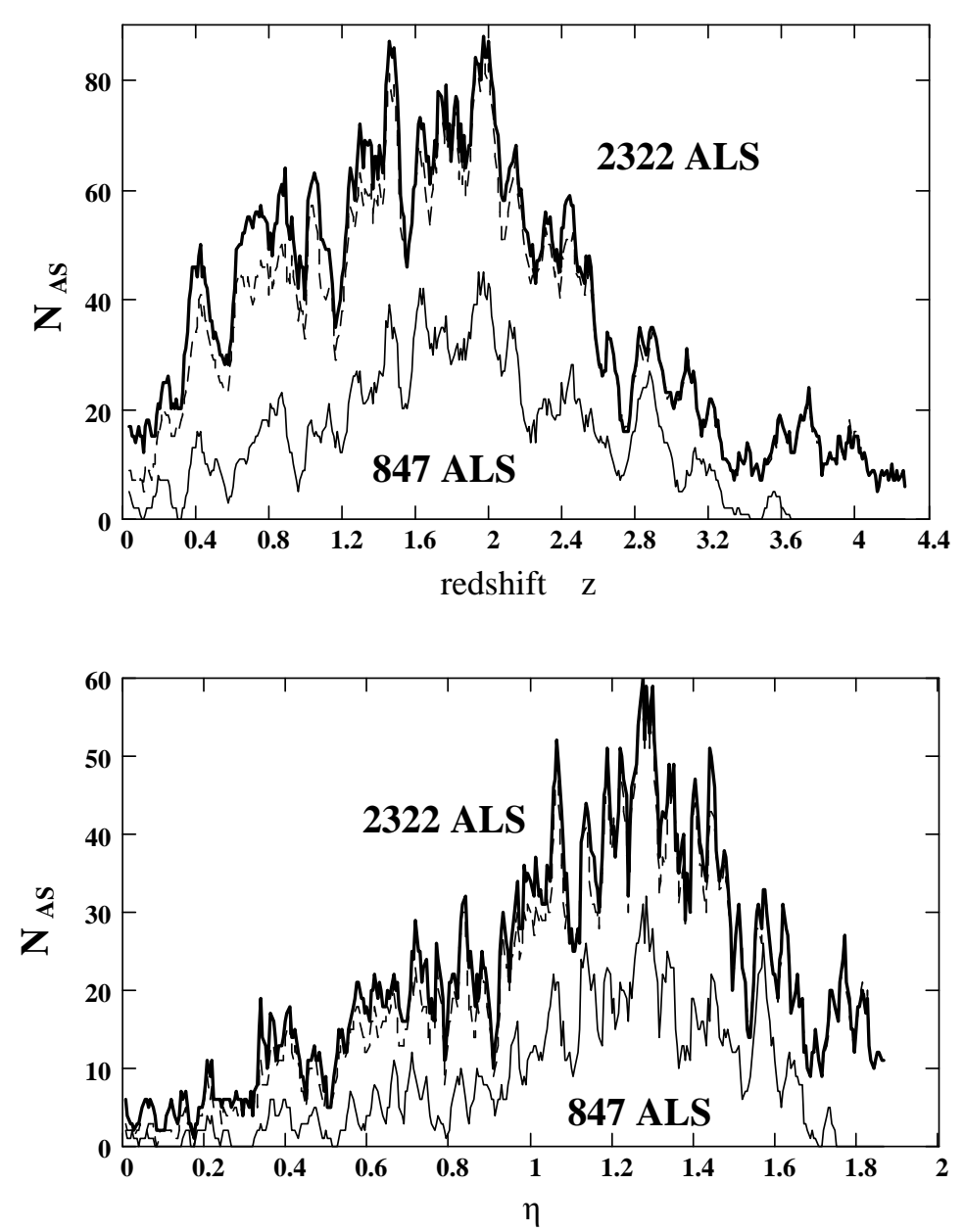

Fig. 1 Upper panel: Sliding-average $z$-distributions of absorption-line systems (ALSs) observed in QSO spectra within the redshift interval $z=0.0-4.30$ with an averaging bin $\Delta_{\mathrm{z}}=0.071$ and a step of its consecutive shift $\delta_{\mathrm{z}}=0.01$; a velocity interval of ALS associations (along each line of sight) into a single system $z_{i}$ (see text) is chosen as $\delta v=500 \mathrm{~km} \mathrm{~s}^{-1}$; thin solid lines -847 systems are taken from the catalog by Junkkarinen et al. (1991), thin dashed lines - 2003 systems - from the catalog by Rvabinkov et al. (2003), thick lines - 2322 systems - from the same but updated catalog. Lower panel: same as in the upper panel but for corresponding $\eta$-distributions (see text) within the appropriate interval $\eta=0.0-1.87$ with an averaging bin $\Delta_{\eta}=0.018$ and a step of shifting $\delta_{\eta}=0.005$.

spectra of 661 QSOs (emission redshifts $z_{e}=0.29-4.9$ ) from the catalog by Rvabinkov et al. (2003). The third one is the $z$-distribution of 2322 ALSs registered in the spectra of 730 QSOs $\left(z_{e}=0.035-5.01\right)$ from the same but updated catalog. All three samples of ALSs represent the same types of metallic systems including single lines or resonance doublets of ions: C II-IV, Mg II, Si II-IV, N V, Al II-III, Fe II etc. They exclude ALSs consisting only of neutral hydrogen lines as well as containing damped Ly $\alpha$ absorption systems (DLA).

Following to Kaminker et al. (2000) we use the procedure of smoothing away an initial sample of ALS redshifts to reveal possible large scale variations and suppress small scale clustering of the ALSs. All redshifts $z_{j}$ registered in a spectrum of a certain QSO and fallen into the velocity interval $\delta v=500 \mathrm{~km} \mathrm{~s}^{-1}$ are treated as a single absorption system with an averaged redshift $z_{a}=\left(\sum_{j=1}^{n_{a}} z_{j}\right) / n_{a}$, where $n_{a}$ is a number of redshifts included in the group. Three distributions in the upper panel of Fig. 1 are obtained using so-called slidingaverage approach which represents a set of consecutive displacements of the averaging bin $\Delta_{\mathrm{z}}=0.071$ along $z$-axis with a step $\delta_{z}=0.01$.

The lower panel of Fig. 1 represents the same three distributions but calculated relatively to a dimensionless line-of-site comoving distance $\eta(z)$ (e.g., Harrison 1993, Kayser et al. 1997, Hogg 1999):

$\eta\left(z_{i}\right)=\int_{0}^{z_{i}} \frac{1}{\sqrt{\Omega_{\mathrm{m}}(1+z)^{3}+\Omega_{\Lambda}}} \mathrm{d} z$,

where $i$ is a numeration of all ALSs $i=1,2, \ldots \mathrm{N}_{\text {tot }}$ sampled as described above, $\mathrm{N}_{\text {tot }}=847,2003$, and 2322, 
respectively. We use the $\Lambda$ CDM-cosmological model with the dimensionless density parameters $\Omega_{\mathrm{m}}=0.23$ and $\Omega_{\Lambda}=1-\Omega_{\mathrm{m}}=0.77$.

A comparison of the $z$-distributions reveals similar patterns of the peaks and dips with rather small $(\sim 15-20 \%)$ amplitude of variations relatively to smoother dependences. The positions of the majority of peaks and dips remain the same after the extension of statistics and some of them become more significant (see Paper I). We have similar situation with $\eta$ distributions, although there is noticeable lack of statistics at $\eta \lesssim 0.6$.

The main accent of the present paper is placed on a search for a periodicity of the ALSs radial distribution with respect to the variable $\eta$. In Section 2 we focus on the periodicity of the $\eta$-distribution. In order to avoid sensitivity of our results to the choice of an averaging bin and to the procedure of a trend elimination, which are mutually dependent, we employ in Sections 2 and 4 a point-like statistical technique. In Section 3 we examine the periodicity of the one-dimensional correlation function calculated in the comoving CS. In Section 4 we estimate a trace of the same periodicity in the distribution of resonance absorption doublets Mg II based on the data of the Sloan Digital Sky Survey (SDSS). Conclusions and discussion of the results in application to the Large-Scale Structure are represented in Section 5. Two toy models for partly ordered simple-cubic lattice displaying similar periodicities are discussed in Appendix.

\section{Periodicity of $\eta$-distribution}

In this Section we employ so called point-like statistical approach at which a sequence of points $\eta_{i} \equiv \eta\left(z_{i}\right)$ calculated with use of Eq. (11) is analysed. To sample ALSs for this analysis we use hereafter the same averaging velocity interval $\delta v=500 \mathrm{~km} \mathrm{~s}^{-1}$ for the association of ALSs into a single redshift $z_{i}$ as in Fig. 1, Additionally, we exclude all ALSs with redshifts $z_{a}$ belonging to a region associated with their host QSO, i.e., at $\left|z_{e}-z_{a}\right| /\left(1+z_{e}\right) \leq \Delta v / c$, where $z_{e}$ is the emission redshift of QSOs and $\Delta v$ is the minimal velocity shift (along a line of sight) adopted for the sample of ALSs. In this paper we choose $\Delta v=1100 \mathrm{~km} \mathrm{~s}^{-1}$, thus the actual sample for our analysis consists of $\mathrm{N}_{\text {tot }}=2167$ systems.

To verify the periodicity of the $\eta$-distribution we calculate a power spectrum for the whole sequence of points $\eta_{i}$ :

$$
\begin{aligned}
\mathrm{P}(\mathrm{k}) & =\frac{1}{\mathrm{~N}_{\mathrm{tot}}}\left\{\left[\sum_{i=i_{0}}^{\mathrm{N}_{\text {tot }}} \cos \left(\frac{2 \pi \mathrm{k} \eta_{i}}{\hat{\eta}}\right)\right]^{2}\right. \\
& \left.+\left[\sum_{i=i_{0}}^{\mathrm{N}_{\text {tot }}} \sin \left(\frac{2 \pi \mathrm{k} \eta_{i}}{\hat{\eta}}\right)\right]^{2}\right\}
\end{aligned}
$$

where $i_{0} \geq 1$ corresponds to a lowest point of the sequence $\eta\left(z_{i}\right), \hat{\eta}=\eta\left(z_{\mathrm{N}_{\text {tot }}}\right)-\eta_{i_{0}}$ is the whole interval under consideration, $\mathrm{k}$ is a harmonic number. A periodicity yields a peak in the power spectrum $\mathcal{P}=\max$ $(\mathrm{P}(\mathrm{k}))$ with a confidence probability

$\beta=[1-\exp (-\mathcal{P})]$,

which is defined with respect to the hypothesis of the Poisson distribution of $\eta_{i}$.

Following Eq. (2) we performed additionally numerous calculations of the power spectra $\mathrm{P}(\mathrm{k})$ for different realizations of randomly distributed points $x_{i}$ $\left(1 \leq i \leq \mathrm{N}_{\text {tot }}\right)$ within intervals $\mathrm{D} x$. We assumed that density of points $x_{i}$ obey to Gaussian distribution with equal values of an expectation and a variance determined by a trend (smooth dependence). Using Kolmogorov criterion we obtained appropriate agreement between distributions of peak values $\mathcal{P}$ at various $\mathrm{k}$ and the exponential distribution. These simulations confirm that a significance probability at certain critical levels $\mathcal{P}_{\text {cr }}$ may be estimated as

$\mathbf{P}\left\{\mathcal{P}(\mathrm{k})>\mathcal{P}_{\text {cr }}\right\}=\exp \left\{-\mathcal{P}_{\text {cr }}\right\}$,

and Eq. (3) does for estimations. Our simulations have shown that the point-like analysis may be treated as the limit case of the standard approach with successively narrowed independent bins. The presence of the trend is a source of most powerful long-waved harmonics (smallest $\mathrm{k}$ ) in the power spectra which should be rejected in our consideration.

The power-spectrum analysis of the ALSs is carried out with using Eq. 2 for successively reduced intervals $z_{\text {min }} \leq z \leq 4.3\left(\eta_{\min } \leq \eta \leq 1.87\right)$, the low boundary being shifted upward from $z_{\min }=0$ to $z_{\min }=2.0$ with a step $\delta z_{\min }=0.01$. Filled regions in the upper panel of Fig. 2 indicate positions of the main peaks with significance $\geq 3 \sigma$ on the $\left(z_{\min }, \mathrm{k}\right)$-plane at integer harmonic numbers. All marked peaks correspond to the same pe$\operatorname{riod} \Delta \eta=\left(\eta_{\max }-\eta_{\min }\right) / \mathrm{k}=0.036 \pm 0.002$. The lower panel displays the value of peaks $\mathrm{P}_{\max }(\mathrm{k})$ calculated for all numbers $\mathrm{k}$ corresponding to the filled regions on the upper panel. Note the presence of periodicity in the $\eta$-distribution of ALSs for the whole interval of $z=0.0$ $-4.3(\eta=0.0-1.87)$ at the significance level $\gtrsim 3 \sigma$. 

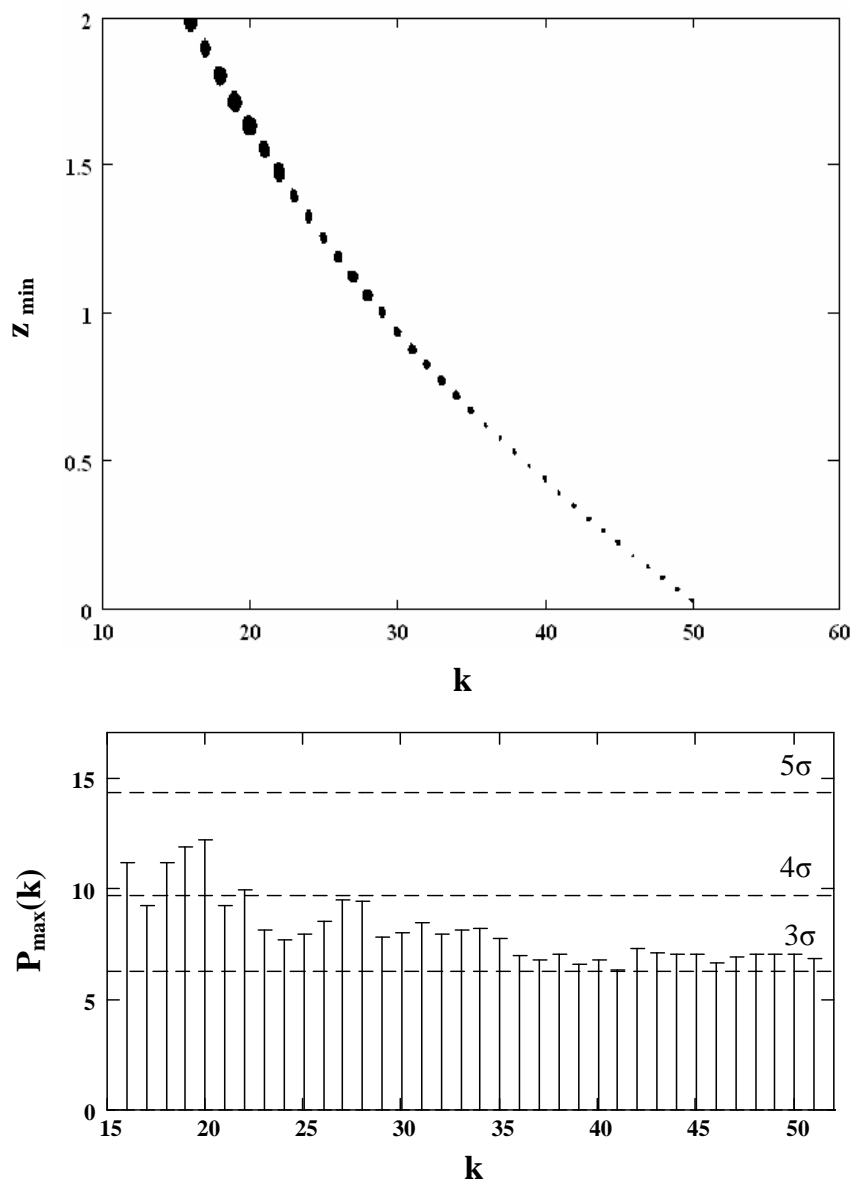

Fig. 2 The set of peaks of the power spectra $\mathrm{P}_{\max }(\mathrm{k})$ calculated according to Eq. (2) in a wide region of harmonic numbers $\mathrm{k}$ at the significance $\geq 3 \sigma$; intervals of $z$ (or $\eta$ ) are limited from below by $z_{\min }\left(\eta_{\min }\right)$ shifted successively upward along an interval $0.0 \leq z_{\min } \leq 2.0$ with a step $\delta z_{\min }=0.01$. The whole sample contains 2167 values $\eta_{i}$ (see text). All visible peaks correspond to the same period $\Delta \eta=0.036 \pm 0.002$. Upper panel indicates positions of the main peaks on the $\left(z_{\mathrm{min}}, \mathrm{k}\right)$-plane; dimensions of filled regions qualitatively denote the values (amplitudes) of the peaks, smallest regions (points) symbolize $3 \sigma$ significance level. Lower panel plots the values of the highest-peaks $\mathrm{P}_{\max }(\mathrm{k})$ versus the integer number $\mathrm{k}$ corresponding to the regions marked on the upper panel. The horizontal dash lines specify the significance levels $3 \sigma, 4 \sigma$, and $5 \sigma$ calculated with use of Eq. (3)

The highest peaks $(\sim 4.5 \sigma)$ take place for reduced intervals $z_{\min } \geq 1.64\left(\eta_{\min } \geq 1.148\right)$ where statistics is essentially better.

For illustration, the upper panels in Figs. 3 and 4 demonstrate the power spectra $\mathrm{P}(\mathrm{k})$ calculated in a wide region of the harmonic number $\mathrm{k}$ for the whole interval $z=0.0-4.30(\eta=0.0-1.87)$ and for reduced one $z=1.64-4.30(\eta=1.148-1.87)$, respectively. The lower panel in Fig. 3 displays a relatively sharp dependence of the main peak amplitudes $\mathrm{P}(\mathrm{k}=51)$ on the density parameter $\Omega_{\mathrm{m}}$, which could prevent one to bring out the period $\Delta \eta$ in the whole interval of $\eta$, e.g., at $\Omega_{\mathrm{m}} \sim 0.3$ (used in Paper I). We assume that this sharp dependence is bounded with a lack of statistics at $\eta \lesssim 0.6$, although it should be verified in further analysis.
The peak at $\mathrm{k}=20(\Delta \eta=0.036)$ in the upper panel of Fig. 4 exceeds the significance level $4.5 \sigma$. The lower panel demonstrates the dependence of the peak amplitude on $\Omega_{\mathrm{m}}$. One can see that in contrast with the lower panel in Fig. 3 the peak values become almost independent of $\Omega_{\mathrm{m}}$ at $\Omega_{\mathrm{m}} \gtrsim 0.2$.

\section{One-dimensional correlation function}

For verification of the periodicity obtained in Section 2 we calculate additionally a two-point correlation function $\xi(\delta \eta)$ for the sample of ALSs. In our consideration a variable $\delta \eta$ substitutes the comoving distance $r$ between pairs of sampled objects in the standard two-point correlation function $\xi(r)$ (e.g., 

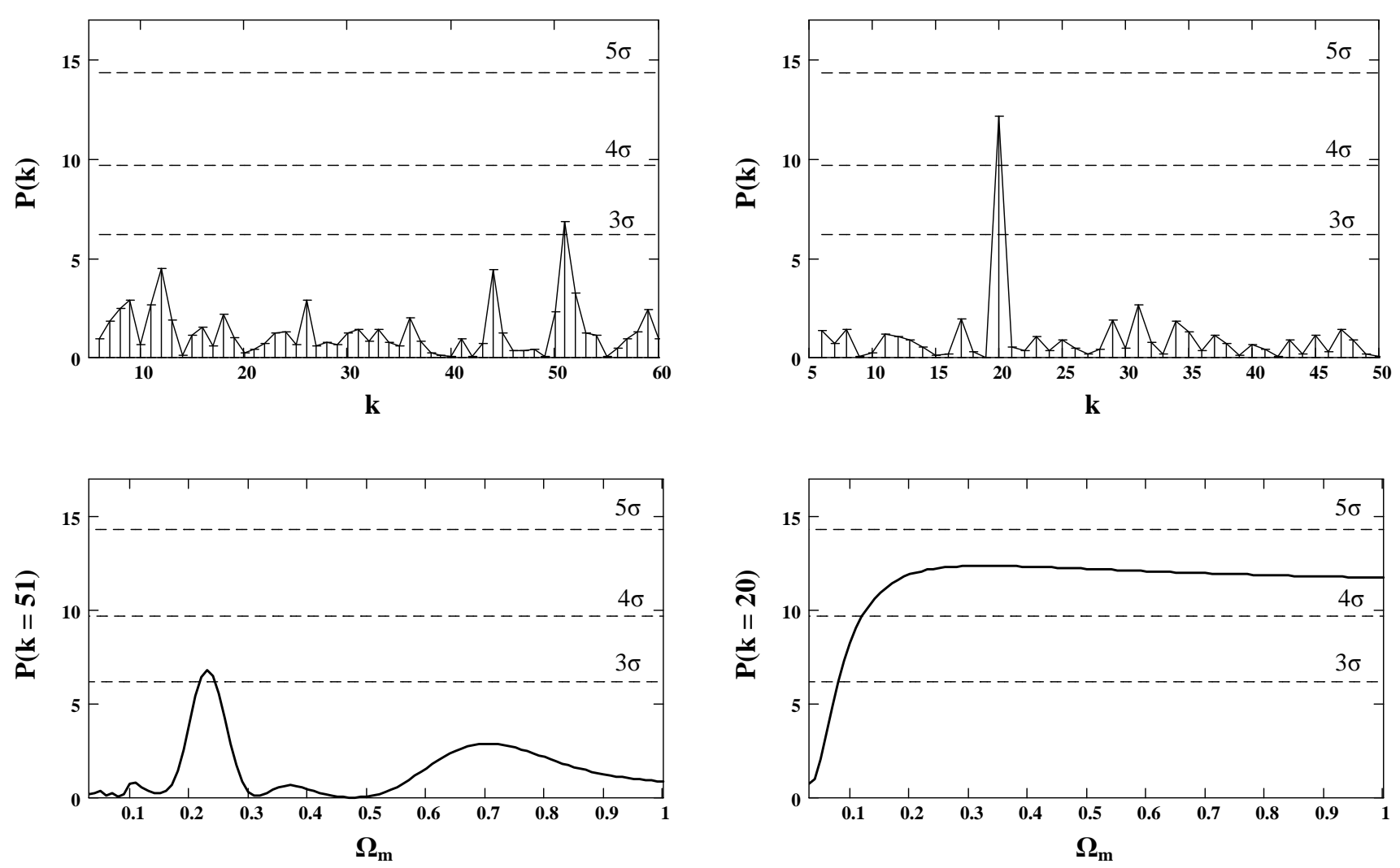

Fig. 3 Upper panel represents the power spectra $\mathrm{P}(\mathrm{k})$ calculated for the whole interval $\eta=0.0-1.87 \quad(z=0.0-4.30)$ at $\Omega_{\mathrm{m}}=0.23$; the main peak corresponds to $\mathrm{k}=51$ and $\Delta \eta=0.037$. Lower panel plots the dependence of the peak value $\mathrm{P}(\mathrm{k}=51)$ on $\Omega_{\mathrm{m}}$.

Davis \& Peebles 1983, Rivolo 1986, Mo et al. 1992, Peebles 1993, Landy \& Szalay 1993, Hamilton 1993, Kerscher et al. 2000, and references therein). Unlike the standard approach the function $\xi(\delta \eta)$ is based on the choice of the single reference center $\eta=0(z=0)$ associated with the observer. Accordingly, all radial (line-of-site) points $\eta_{i}\left(z_{i}\right)$ within a layer $\left(\eta \pm \Delta_{\eta} / 2\right)$, where $\Delta_{\eta}$ is a width of bin, are treated as equivalent ones despite of various spatial distances between them.

In this way we count up all pairs with fixed relative line-of-site comoving distance $\delta \eta_{i, j}=\left|\eta_{i}-\eta_{j}\right|$ between arbitrary ALSs numerated by $i$ and $j$, which are registered in various directions. Thus one can write:

$\xi(\delta \eta)=\frac{\mathcal{N}_{\text {obs }}(\delta \eta)}{\mathcal{N}_{\text {sim }}(\delta \eta)}-1$,

where $\mathcal{N}_{\text {obs }}(\delta \eta)$ is a number of observed pairs of ALSs separated by $\delta \eta=\delta \eta_{i, j}$ belonging to the interval $\delta \eta \pm \Delta_{\eta} / 2$. The bin width is chosen as $\Delta_{\eta}=0.018$, i.e., the same as in low panel of Fig. 1. $\mathcal{N}_{\text {sim }}(\delta \eta)$ is a number of crossing pairs between the real sample of ALSs and the points of a random (Poisson) sample sim-

Fig. 4 Same as in Fig. 3 but for the most significant peak $(4.5 \sigma)$ in Fig. 2 Upper panel represents the power spectrum $\mathrm{P}(\mathrm{k})$ calculated for the interval $\eta=1.148-1.87 \quad(z=1.64-$ $4.30) \quad$ at $\Omega_{\mathrm{m}}=0.23$; the sample contains $\mathrm{N}_{\mathrm{AS}}=1148$ ALSs. Lower panel plots the dependence of the main peak $\mathrm{P}(\mathrm{k}=20)$ on $\Omega_{\mathrm{m}}$.

ulated in the same interval of $\eta$ with the same smoothed distribution (trend) as the real sample. The both samples (real and simulated ones) have the same number of redshift points.

Note also that the correlation function (5) differs from the two-point multicentric correlation function which has been calculated for both radial (line-ofsight) and transverse directions by Gaztañaga et al. $(2009 \mathrm{a}, \mathrm{b})$ for the spatial distribution of galaxies (see also Kazin et al. 2010b). On the other hand, the function (5) differs from the correlation functions calculated by counting pairs of objects along separate lines of sight (e.g., Quashnock et al. 1996, Broadhurst \& Jaffe 2000), although the results of both approaches seem to be compatible.

The upper panel of Fig. 5 displays the one-dimensional (two-point) correlation function (5) calculated in units of the appropriate Poisson error $\sigma(\xi)=(1+$ $\xi) / \sqrt{\mathcal{N}_{\text {sim }}(\delta \eta)}$ (e.g., Peacock \& Nicholson 1991):

$\frac{\xi(\delta \eta)}{\sigma(\xi)} \approx \frac{\mathcal{N}_{\text {obs }}(\delta \eta)-\mathcal{N}_{\text {sim }}(\delta \eta)}{\sqrt{\mathcal{N}_{\text {sim }}(\delta \eta)}}$ 

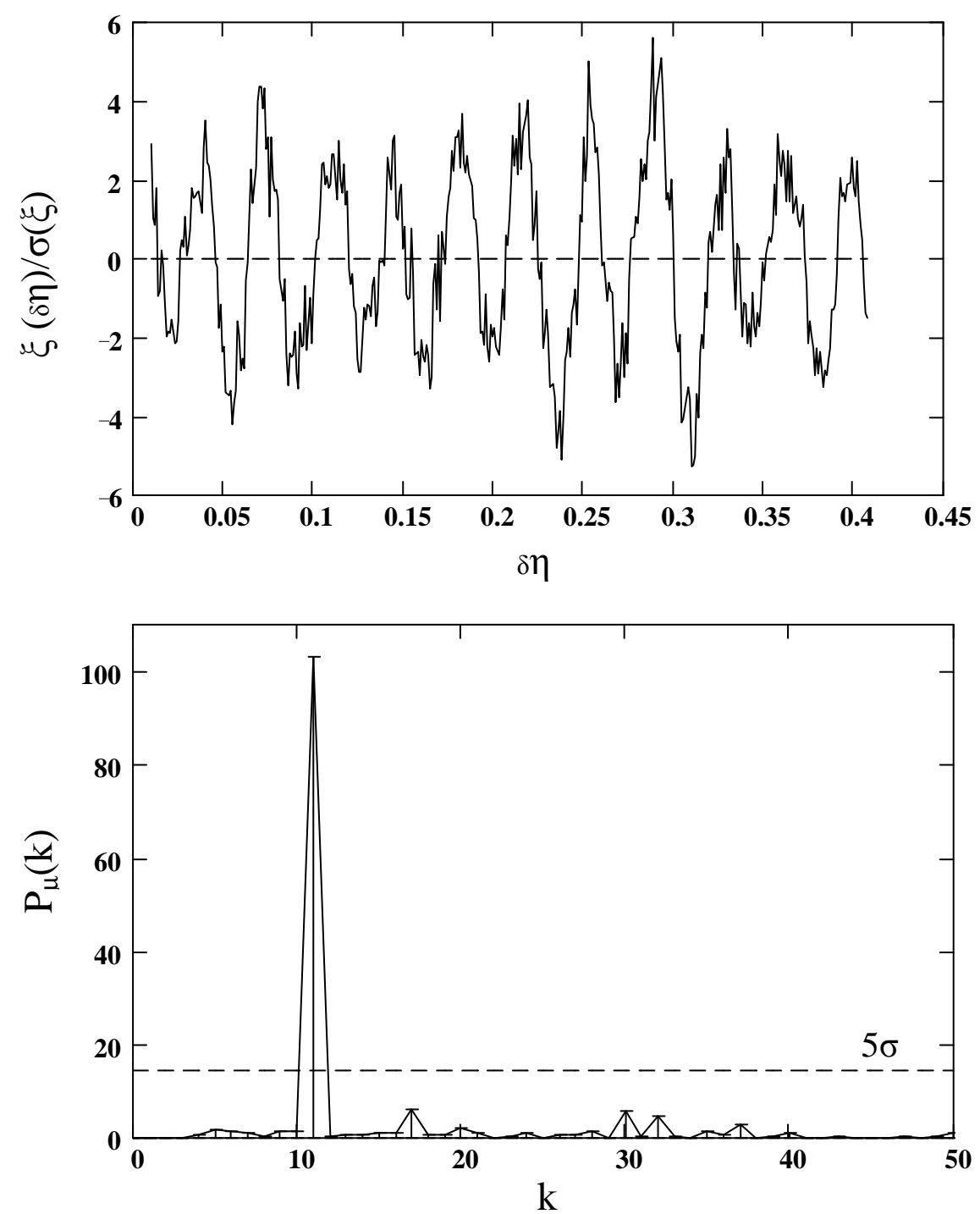

Fig. 5 Upper panel: two-points correlation function $\xi(\delta \eta) / \sigma(\xi)$ (see text) versus the interval of $\delta \eta(0.01 \leq \delta \eta \leq 0.408)$ between the components of pairs constituted from the same sample of 1148 ALSs as in Fig. 4 Lower panel: power spectrum $\mathrm{P}_{\mu}(\mathrm{k})$ calculated for $\mu=\mu(\eta)=\xi(\delta \eta) / \sigma(\xi)$ according to Eq. [7), $\mathrm{k}$ is the harmonic number.

An interval of values $\delta \eta$ in Fig. 5 ( $0.01 \leq \delta \eta \leq$ $0.408)$ is chosen about twice shorter than the $\eta$-interval $(1.148 \leq \eta \leq 1.870)$ to provide its uniform filling by various pairs of ALSs. One can see the sequence of positive and negative peaks with the significance $\gtrsim 3 \sigma$ with respect to zero level. Let us notice the presence of a long-range order in the dependence of $\xi(\delta \eta) / \sigma(\xi)$ on $\delta \eta$. We discuss this effect in Section 5 .

The lower panel in Fig. 5 represents the power spectrum calculated for the value $\mu=\mu(\delta \eta)=\xi(\delta \eta) / \sigma(\xi)$ according to the equation:

$$
\begin{aligned}
\mathrm{P}_{\mu}(\mathrm{k}) & =\frac{1}{N_{\mu} \hat{U}(\mathrm{k})}\left\{\left[\sum_{m=1}^{N_{\mu}} \mu_{m} \cos \left(\frac{2 \pi \mathrm{k} \delta \eta_{m}}{\delta \hat{\eta}}\right)\right]^{2}\right. \\
& \left.+\left[\sum_{m=1}^{N_{\mu}} \mu_{m} \sin \left(\frac{2 \pi \mathrm{k} \delta \eta_{m}}{\delta \hat{\eta}}\right)\right]^{2}\right\}
\end{aligned}
$$

where $\mu_{m}=\mu\left(\delta \eta_{m}\right)$, the values $\delta \eta_{m}$ run over a set of points $m=1,2, \ldots N_{\mu}$ of the variable $\delta \eta$ from 0.01 to 0.408 . Here we employ the sliding bins $\Delta_{\eta}=0.018$ with centers at $\delta \eta_{m}$ which transit from one center to another one by successive shifts $h_{\eta}=0.001 ; N_{\mu}=399$ is the number of the points $\delta \eta_{m}$ within the whole interval 
$\delta \hat{\eta}=0.398$. The value $\hat{U}(\mathrm{k})$ in the denominator of (7) may be presented as

$\hat{U}(\mathrm{k})=\left\{\begin{array}{ccc}U(\mathrm{k}) & \text { at } & U(\mathrm{k})>1 \\ 1 & \text { at } & U(\mathrm{k}) \leq 1\end{array}\right.$

where

$U(\mathrm{k})=\left[\sin \left(\frac{\pi \mathrm{k} \Delta_{\eta}}{\delta \hat{\eta}}\right) \frac{\delta \hat{\eta}}{\pi \mathrm{k} \Delta_{\eta}}\right]^{2} \frac{\Delta_{\eta}}{h_{\eta}}$.

It is known from spectral analysis (e.g., Bath 1974), that the sliding-average procedure corresponds to a lowfrequency filtration with a rectangular function as a filter. Fourier transform of this function is represented by $\sin (\omega / 2) /(\omega / 2)$, where $\omega$ is a dimensionless Fourier frequency. Note that in the considered case a factor $\Delta_{\eta} / h_{\eta}$ in Eq. (9) is equal to 18. Our special simulations of random catalogs of points have shown that the normalization factor (8), (9) in the denominator of expressions analogous to Eq. (7) compensates a low-frequency distortion through the sliding-average filtration and yields the exponential probability functions given by Eq. (4). Therefore we use Eq. (31) for estimations of the confidence probability of peaks in the power spectrum.

In result we obtain a strong peak of $\mathrm{P}_{\mu}(\mathrm{k})$ at $\mathrm{k}=$ 11 on the significance level well exceeding $5 \sigma$. This peak manifests the periodicity of the correlation function with the period $\Delta \eta=0.036 \pm 0.002$ which is in agreement with the periodicity discussed in Sect. 2

\section{Periodicity in distribution of Mg II systems}

It should be noted in this context that a set of statistical investigations of resonance absorption doublets $\mathrm{Mg}$ II $(\lambda 2796,2803 \AA)$ on the base of SDSS catalog have been performed recently by a few groups (e.g., Bouché et al. 2006, Prochter et al. 2006, Ménard et al. 2008, Lundgren et al. 2009). It follows from their results that the spatial distribution of strong doublet lines $\mathrm{Mg}$ II with $\mathrm{W}_{\mathrm{r}}^{\lambda 2796}>1 \AA$ differs from the distribution of relatively weak lines $\mathrm{W}_{\mathrm{r}}^{\lambda 2796}<1 \AA$, where $\mathrm{W}_{\mathrm{r}}^{\lambda 2796}$ is the rest frame equivalent width of the line $\lambda=2796 \AA$. It seems to be plausible that the strong and weak Mg II absorbers are associated with different galaxy populations which display different evolving (e.g., Lundgren et al. 2009) and morphological (e.g., Kacprzak et al. 2007; 2008) properties. Therefore one could anticipate that radial distributions of both types of absorption systems correspond to different tendencies of clustering and different periodicities. For instance, weaker absorbers appear to be spreaded over space-time more homogeneously and with weaker effects of galaxies evolution.
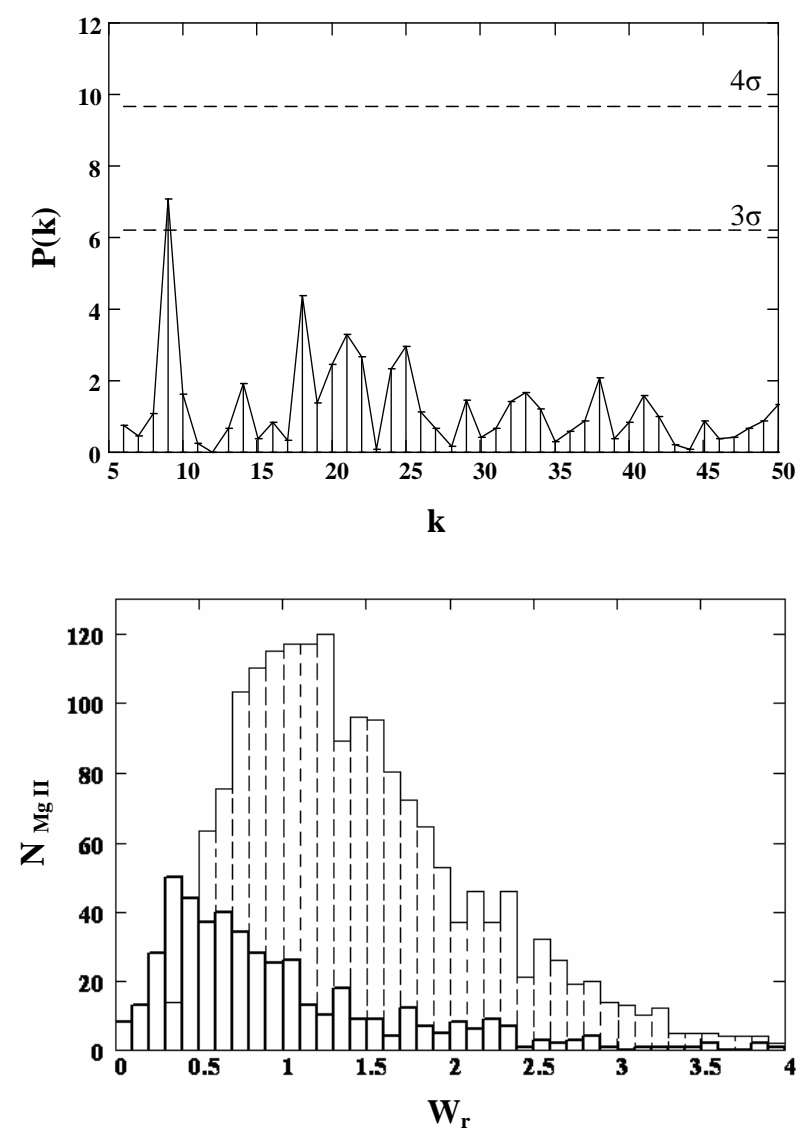

Fig. 6 Upper panel: Power spectrum $\mathrm{P}(\mathrm{k})(5 \leq \mathrm{k} \leq 50)$ calculated in the point-like statistical approach (Eq. (2) ) with selection of 961 absorption systems $\mathrm{Mg}$ II from the catalog by Bouché et al. (2006) within the interval $0.38 \leq z \leq 0.8(0.35 \leq \eta \leq 0.67)$ satisfying a condition of $\mathrm{W}_{\mathrm{r}} \leq 1.4 \AA$. Lower panel: Comparison of two histograms displaying distributions of $478 \mathrm{Mg}$ II absorption systems from the catalog by Rvabinkov et al. (2003) (thick solid lines) and $1806 \mathrm{Mg}$ II systems from the catalog by Bouché et al. (2006) (long dashed lines) with respect to the rest-frame equivalent width $\mathrm{W}_{\mathrm{r}}$.

Let us note that the main result of the present paper is bound up with relatively weak ALSs. It is illustrated on the lower panel of Fig. 6 by the histograms displaying the distribution of $478 \mathrm{Mg}$ II absorption doublets $(\lambda 2796,2803)$ involved in our analysis with respect to $\mathrm{W}_{\mathrm{r}}^{\lambda 2796}$ and the analogous distribution of $1806 \mathrm{Mg}$ II systems from the catalog by Bouché et al. (2006) based on the third data release (DR3) of the SDSS. The distribution of Mg II systems from our catalog has a maximum at $\mathrm{W}_{\mathrm{r}}^{\lambda 2796} \lesssim 0.4 \AA$ while the distribution of the Mg II absorbers from the catalog of Bouché et al. (2006) shows a maximum at $\mathrm{W}_{\mathrm{r}}^{\lambda 2796} \gtrsim 1.0 \AA$, i.e., the former one is noticeably shifted towards lower values of $\mathrm{W}_{\mathrm{r}}^{\lambda 2796}$. Let us note that the more extended catalogue of Mg II systems by Prochter et al. (2006) also 

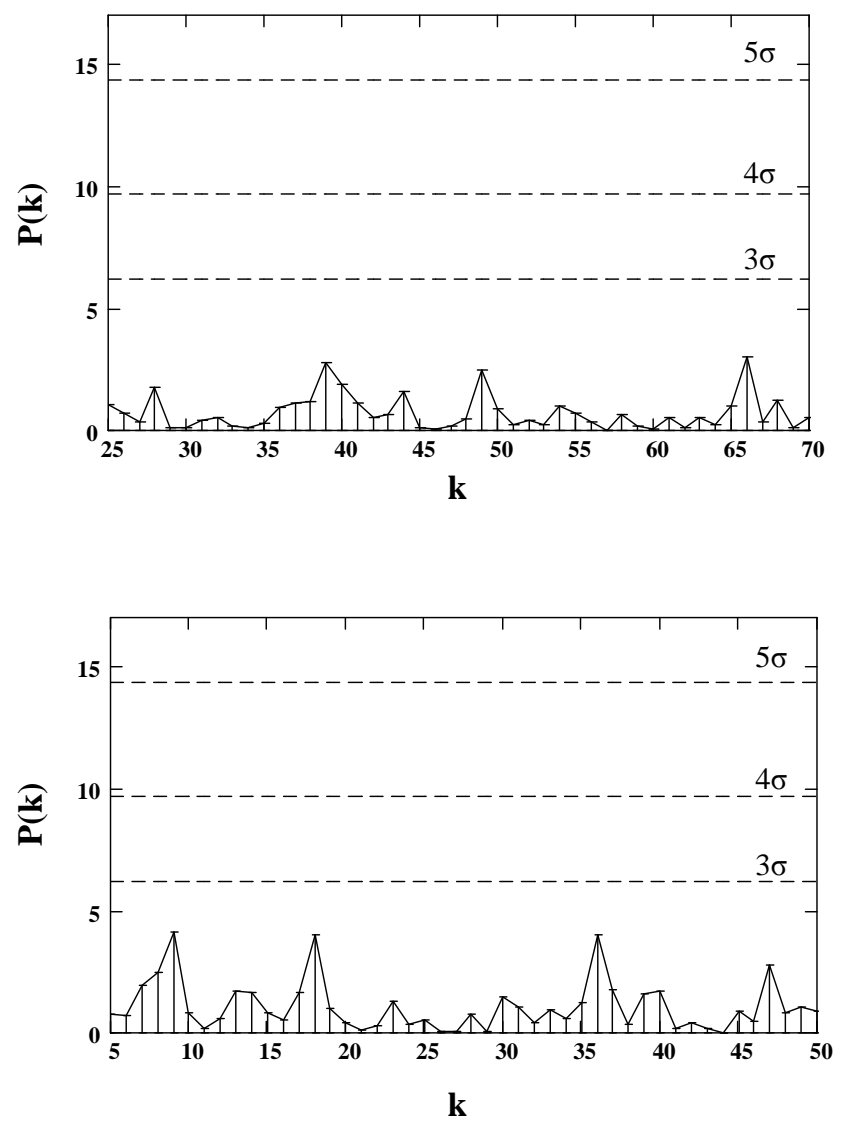

Fig. 7 Upper panel: Power spectrum $\mathrm{P}(\mathrm{k})$ calculated with using Eq. (2) for 730 QSOs $\left(0.035 \leq z_{e} \leq 5.01\right)$ from the catalogue Rvabinkov et al. (2003), whose spectra include 2322 ALSs used in the present analysis. An interval of harmonic numbers $25 \leq \mathrm{k} \leq 70$ corresponds to comoving scales (233.383.3) $h^{-1} \mathrm{Mpc}$, at $\Omega_{\mathrm{m}}=0.23$. Lower panel: Same as the upper panel but for 961 QSOs $\left(0.42 \leq z_{e} \leq 2.90\right)$ from the catalogue Bouché et al. (2006), whose spectra include 961 systems of $\mathrm{Mg}$ II used for the calculations of $\mathrm{P}(\mathrm{k})$ in Fig. 6 An interval of harmonic numbers $5 \leq \mathrm{k} \leq 50$ corresponds to comoving scales $(712.9-71.3) h^{-1} \mathrm{Mpc}$.

based on SDSS data accumulates still stronger lines with $\mathrm{W}_{\mathrm{r}}^{\lambda 2796} \geq 1 \AA$ which are still less consistent with our sample of ALSs. As discussed above it is likely that these two samples correspond predominantly to different populations of galaxies.

Nevertheless, we estimate here the presence of the same periodicity in the $\eta$-distribution of $\mathrm{Mg}$ II systems as discussed in Sections 2 and 3 restricting ourselves by relatively weak systems $\left(\mathrm{W}_{\mathrm{r}}^{\lambda 2796} \lesssim 1 \AA\right)$. The upper panel in Fig. 6 represents the power spectrum $\mathrm{P}(k)$ calculated according to the point-like approach of Eq. (2). We employ $\mathrm{N}_{\text {tot }}=961$ systems from the cata$\log$ of Bouché et al. $(2006)$ at $\mathrm{W}_{\mathrm{r}}^{\lambda 2796} \leq 1.4 \AA$ within the interval $0.35 \leq \eta \leq 0.67(0.38 \leq z \leq 0.80)$. One can see the peak $\mathrm{P}(k)$ at $k=9$ for the same period $\Delta \eta=\left(\eta_{\max }-\eta_{\min }\right) / k=0.036 \pm 0.002$ as in Figs. 2 5 .

Additionally, we test possible selection effects which might induce periodicities in the initial samples of QSOs. Examples of such simulated periodicities are given, e.g., by Hartnett (2009) and Bell \& Comeau (2010). The upper panel in Fig. 7 represents the power spectrum $\mathrm{P}(\mathrm{k})$ calculated according to Eq. (2) for 730 original QSOs within an interval of $\eta_{e}=0.035-1.98$ $\left(z_{e}=0.035-5.01\right)$ sampled for the statistical treatment of 2322 ALSs. We see that in the chosen range of harmonic numbers, $\mathrm{k}=25-70$, there are no significant peaks in the power spectrum of the QSOs. Note that the period $\Delta \eta=0.036$ indicated in Sections 2 and 3 corresponds to the harmonic number $\mathrm{k}=54$. The lower panel demonstrates similar power spectrum calculated for 961 QSOs within an interval of $\eta_{e}=0.39-1.58$ $\left(z_{e}=0.42-2.90\right)$ from the catalog by Bouché et al. (2006). We use their spectra for the test of periodicity of 961 relatively weak $\left(\mathrm{W}_{\mathrm{r}} \leq 1.4 \AA\right)$ systems $\mathrm{Mg}$ II. The power spectrum also does not contain significant peaks. Note that the period $\Delta \eta=0.036$ corresponds to the harmonic number $\mathrm{k}=33$. On the other hand, possible simulated periodicities at $\Delta z_{e}=0.258,0.312$, and 0.44 in $z_{e}$-distribution of QSOs indicated by Hartnett (2009) for SDSS data correspond to harmonic numbers $\mathrm{k} \approx 10,8$, and 6 , respectively. Additional analysis of possible selection effects was carried out in Paper I.

\section{Conclusions and discussion}

On the base of the updated catalog of absorption systems (Ryabinkov et al. 2003) we have performed the statistical analysis of 2322 absorption-line systems (ALSs) detected in the QSO spectra in the redshift range $z=0.0-4.3$. The ALS redshifts are averaged within the velocity interval $\delta v=500 \mathrm{~km} \mathrm{~s}^{-1}$ along each line of sight. This velocity interval containing one averaged point corresponds to the spatial scales $\sim 5-6 h^{-1} \mathrm{Mpc}$ (at $z=0.0-4.3$ ) of comoving distances for $\Lambda \mathrm{CDM}$ model at $\Omega_{\mathrm{m}}=0.23$. The averaged points can trace possible large-scale variations of absorbing matter.

The main results can be summarized as follows:

(1) The distribution of the ALSs relative to the dimensionless comoving distances $\eta(z)$ given by Eq. (11) displays a statistically significant pattern of alternating maxima (peaks) and minima (dips) against a smoother curve (trend). This sequence of peaks and dips comprises a certain periodical component with a period of $\Delta \eta=0.036 \pm 0.002$. The power spectrum calculated according to Eq. (2) for the whole interval of $\eta(\eta=0.0$ 
- 1.87) contains the peak at the significance level exceeding $3 \sigma$ relatively to the hypothesis of the Poisson $\eta$-distribution. In the most statistically representative interval $\eta=1.148-1.870(z=1.64-4.3)$ the same periodicity reveals itself at the significance level $4.5 \sigma$. Still more prominent peak corresponding to the same period $\Delta \eta$ arises in the power spectrum calculated for the twopoint correlation function $\xi(\delta \eta)$ with use of Eqs. (7) 9).

(2) The same period has been revealed also in the power spectra of $961 \mathrm{Mg}$ II $(\lambda 2796,2803)$ absorption systems from the catalog by Bouché et al. (2006) within the interval $0.35 \leq \eta \leq 0.67(0.38 \leq z \leq 0.80)$ calculated for relatively small rest frame equivalent widths $\mathrm{W}_{\mathrm{r}}^{\lambda 2796} \leq 1.4 \AA$. Although the appropriate peak in the power spectra is not very significant (slightly exceeds $3 \sigma)$ this is comparable with the peak obtained in Section 2 for the sample of ALSs within the whole interval $\eta=0.0-1.87(z=0.0-4.3)$. It may evidence for availability at least a weak periodical component at $z<1$.

(3) The special feature of the results is the appearance of the long-range (or intermediate-range) order visually displayed by the two-point correlation function in Fig. 5]. This order corresponds to some stability of the phase of periodical component which leads to an invariance of the correlation function under translations by a distance multiple of the period $\Delta \eta$. The significant peak at the lower panel in Fig. 5 may be treated as a measure of such an order.

(4) The dimensionless period $\Delta \eta$ obtained here corresponds to the spatial scale of line-of-sight comoving distance $D_{\mathrm{c}}=c / H_{0} \times \Delta \eta=108 \pm 6 h^{-1}$ Mpc. According to Table 1 this scale $D_{\text {c }}$ is consistent with a characteristic scale of the Large Scale Structure (LSS) in the spatial distribution of galaxies and clusters of galaxies at relatively small redshifts $z<0.5$. On the other hand, the appropriate temporal interval $T_{\mathrm{c}}=1 / H \times \Delta \eta=$ $350 \pm 20 h^{-1} \mathrm{Myr}$ is consistent with recent results of Aref'eva \& Koshelev (2008).

Summarized results admit both interpretations of the periodicity: spatial one - appearance of partly ordered spatial structures of matter in the early Universe (see below) or temporal one - generation of some temporal wave process in the course of the cosmological evolution. For instance, Kaminker et al. (2000) and Paper I argued rather in favour of the temporal interpretation of the features of ALS distribution. In this paper, however, we follow a spatial consideration. Note that both hypotheses have to obey to the cosmological principle (e.g, Peebles 1993), i.e., partly ordered structures or periodical temporal process could be observed in any spatial points of the Universe.

A special wave of interest to the effects of periodicity in the redshift distribution of galaxies was initi- ated by Broadhurst et al. (1990). Their pencil-beam surveys near the Galactic poles displayed a periodicity on a scale about $130 h^{-1} \mathrm{Mpc}$ which might be interpreted either as a pure spatial quasi-periodic pattern of LSS constituents (set of clumps or walls and voids; e.g., Kurki-Suonio et al. 1990, Kaiser \& Peacock 1991, van de Weygaert 1991, Dekel et al. 1992, Yoshida et al. 2001 and references therein) or as a spatial-temporal sequence of pronounced and depressed epochs which could become apparent in the distribution of matter (e.g., Morikawa 1991, Hirano et al. 2008).

Table 1 represents the examples of characteristic scales obtained in last years by a few groups of authors employing statistical analysis of galaxies and clusters of galaxies. The characteristic scales in the last but one column may be approximately subdivided into two groups: the scales belonging to the interval $70 \pm 20 h^{-1} \mathrm{Mpc}$ and the scales within $120 \pm$ $20 h^{-1}$ Mpc. The period $D_{\text {c }}=108 \pm 6 h^{-1} \mathrm{Mpc}$ gets into the latter group. Especially good consent occurs with results obtained by Percival et al. (2007a), Gaztañaga et al. (2009a) and Kazin et al. (2010a) for the characteristic scale of the Baryon Acoustic Oscillations (BAOs) in the large-scale distribution of matter (see also Martínez et al. 2009).

Actually, the BAOs are discussed in literature in connection with a scale of the sound horizon at the epoch of recombination (e.g., Blake \& Glazebrook 2003, Percival et al. 2007b) which may be measured as a single peculiarity (bump) in the spatial correlation function (e.g., Eisenstein et al. 2005). On the other hand, this spatial scale manifests itself as a series of regular variations imprinted on the power spectrum of the distribution of galaxies (e.g., Eisenstein \& Hu 1998, Eisenstein et al. 1998, Percival et al. 2007b). By contrast, we discuss here the regular spatial variations providing one prominent peak in the power spectra. In this context let us note that the correlation function calculated by Martínez et al. (2009) shows a hint of a possible secondary peak at a scale of $\sim 170 h^{-1}$ Mpc. Being confirmed such a tendency would be in consent with the hypothesis that at least an intermediate-range order may be present in the spatial distribution of ALSs.

Still more pronounced regular spatial variations of the standard (see Section 3) two-point correlation function of galaxy superclusters were obtained, e.g., by Einasto et al. (1997a) and Tago et al. (2002). These authors indicated the possibility of a long-range (or intermediate-range) order with a characteristic period of $115 \pm 7 h^{-1} \mathrm{Mpc}$ in the spatial distribution of superclusters: the largest relatively isolated bounded systems. Their results are qualitatively consistent with the two-point correlation function shown in Fig. 5. 
Table 1 Characteristic scales of LSS relatively to comoving system in $\Lambda$ CDM-model

\begin{tabular}{|c|c|c|c|c|c|}
\hline Objects & $\begin{array}{l}\text { Surveys } \\
\text { Catalogs }\end{array}$ & Redshifts & $\begin{array}{c}\Lambda \mathrm{CDM} \\
\Omega_{\mathrm{m}}\end{array}$ & $\begin{array}{c}\text { Scales } \\
h^{-1} \mathrm{Mpc}\end{array}$ & References \\
\hline \multirow[t]{2}{*}{$92,952 \mathrm{G}^{1)}$} & SDSS DR1 ${ }^{5)}$ (radial) & $z<0.14$ & & $74 \pm 17$ & \\
\hline & & & 0.3 & & 14) \\
\hline $16,756 \mathrm{G}$ & LCRS ${ }^{6)}$ & $z<0.11$ & & $60 \pm 10$ & \\
\hline $1324 \mathrm{CG}^{2)}$ & Abell CG & & & & \\
\hline+ & + & $z<0.28$ & 11) & $115 \pm 7$ & 15) \\
\hline 284 X-ray $\mathrm{CG}^{3}$ ) & $\operatorname{RBS}{ }^{7)}$ & & & & \\
\hline $229,193 \mathrm{G}$ & $2 \mathrm{dF} \mathrm{GRS}^{8)}$ & & & $73.4 \pm 5.8$ & \\
\hline+ & + & $z<0.3$ & 12) & + & 16) \\
\hline $427,512 \mathrm{G}$ & SDSS DR5 & & & $127 \pm 21$ & \\
\hline $143,368 \mathrm{G}$ & $2 \mathrm{dF}$ GRS & $z=0.2$ & & $112.9 \pm 3.3$ & \\
\hline+ & + & & $0.23^{13)}$ & & 17) \\
\hline \multirow[t]{2}{*}{$77,801 \mathrm{LRG}^{4)}$} & SDSS DR5 & $z=0.35$ & & $104.7 \pm 3.2$ & \\
\hline & & $0.15 \leq z \leq 0.30$ & & $110.3 \pm 3.9$ & \\
\hline \multirow[t]{2}{*}{ 75,000 LRG } & SDSS DR6 & & 0.25 & & 18) \\
\hline & & $0.40 \leq z \leq 0.47$ & & $108.9 \pm 4.0$ & \\
\hline $105,831 \mathrm{LRG}$ & SDSS DR7 & $0.16<z<0.36$ & 0.25 & $101.7 \pm 3.0$ & 19) \\
\hline $2167 \mathrm{ALS}$ & $\mathrm{RKV}^{9)}$ & $0<z \leq 4.3$ & 0.23 & $108 \pm 6$ & present \\
\hline $961 \mathrm{Mg}$ II & Bouché 10) & $0.38 \leq z \leq 0.80$ & 0.23 & $108 \pm 6$ & work \\
\hline
\end{tabular}

1) $\mathrm{G}$ - Galaxies; ${ }^{2)} \mathrm{CG}$ - clusters of galaxies; ${ }^{3)} \mathrm{X}$-ray CG - X-ray clusters of galaxies; ${ }^{4)}$ LRG - Luminous red galaxies; ${ }^{5)}$ SDSS - Sloan Digital Sky Survey; ${ }^{6}$ LCRS - Las Campanas Redshift Survey; ${ }^{7)}$ RBS - ROSAT Bright Survey; ${ }^{8)}$ 2dF GRS - 2dF Galaxy Redshift Survey; ${ }^{9}$ RKV - Rvabinkov et al. (2003); ${ }^{10)}$ Bouché et al. (2006); ${ }^{11)}$ distances between clusters were calculated using the Mattig (1958) formula with the deceleration parameter $q_{0}=0.5$ (Einasto et al. 2001); ${ }^{12}$ ) distance intervals were calculated using $\Delta r=c \Delta z / H_{0} ;{ }^{13)}$ the scales $r_{\mathrm{s}}$ have been recalculated for this Table at $\left.\Omega_{\mathrm{m}}=0.23 ; 14\right)$ Doroshkevich et al. (2004); 15) Tago et al. (2002); 16) Hartnett \& Hirano (2008); 17) Percival et al. (2007a); 18) Gaztañaga et al. (2009a); 19) Kazin et al. (2010a). Sign + means that the data of both catalogs and both types of objects were used, as well as both scales were revealed for the same sample of data.

Einasto et al. (1997b) considered a model of a quasiregular lattice with random distribution of clusters and superclusters along lattice edges. They showed that the spatial correlation function for a partly regular structure displays oscillations similar to those revealed in the correlation function of superclusters.

By analogy with Einasto et al. (1997b) we consider in the Appendix two toy models of partly regular structures with essential random components and calculate the power spectrum of radial distribution of points. The power spectra for both models display the peaks with approximately the same significance as in Fig. 4 It illustrates that rather moderate spatial ordering might yield the periodicity of radial distribution similar to the sample of ALSs discussed here.

It should be emphasized that we represent only visual examples of partly ordered structure without strict arguments in favour of the spatial interpretation of the periodicity obtained. This interpretation should be verified by special investigations in a future.

Acknowledgments We thank A.Y. Potekhin for technical assistance in preparation of the paper. The work has been supported partly by the RFBR (grant No. 0802-01246a), and by the State Program "Leading Scientific Schools of RF" (grant NSh 3769.2010.2). 


\section{References}

Aref'eva I. Ya., \& Koshelev A. S., 2008, JHEP, 9, 68 (arXiv:0804.3570)

Bath M., 1974, Spectral analysis in geophysics, Elsevier Science

Bell M. B., \& Comeau S. P., 2010, Ap\&SS, 326, 11

Blake C., \& Glazebrook K., 2003, Astrophys. J., 594, 665

Broadhurst T. J., Ellis R. S., Koo D. C., \& Szalay A. S., 1990, Nature, 343, 726

Broadhurst T., \& Jaffe A. H., 2000, in Mazure A., Le Févre O., Le Brun V., eds, Clustering at High Redshift, ASP Conference Series, 200, p. 241

Bouché N., Murphy M. T., Péroux C., Csabai I., \& Wild V., 2006, Mon. Not. R. Astron. Soc., 371, 495

Davis M., \& Peebles P. J. E., 1983, Astrophys. J., 267, 465

Dekel A., Blumenthal G. R., Primack J. R., \& Stanhill D., 1992, Mon. Not. R. Astron. Soc., 257, 715

Doroshkevich A. G., Tucker D. L., Allam S., \& Way M. J., 2004, Astron. Astrophys., 418, 7

Einasto J., Einasto M., Frisch P. et al., 1997a, Mon. Not. R. Astron. Soc., 289, 801

Einasto J., Einasto M., Frisch P. et al., 1997b, Mon. Not. R. Astron. Soc., 289, 813

Einasto M., Einasto J., Tago E., Müller V., \& Andernach H., 2001, Astron. J., 122, 2222

Eisenstein D. J., \& Hu W., 1998, Astrophys. J., 496, 605

Eisenstein D. J., Hu W., \& Tegmark M., 1998, Astrophys. J., 504, L57

Eisenstein D. J., Zehavi I., Hogg D. W. et al., 2005, Astrophys. J., 633, 560

Gaztañaga E., Cabré A., \& Hui L., 2009a, Mon. Not. R. Astron. Soc., 399, 1663

Gaztañaga E., Miquel R., \& Sánchez E., 2009b, Rhys. Rev. Lett., 103, 091302

Hamilton A. J. S., 1993, Astrophys. J., 417, 19

Harrison E., 1993, Astrophys. J., 403, 28

Hartnett J. G., \& Hirano K., 2008, Ap\&SS, 318, 13

Hartnett J. G., 2009, Ap\&SS, 324, 13

Hirano K., Kawabata K., \& Komiya Z., 2008, Ap\&SS, 315, 53

Hogg D. W., 1999, preprint (astro-ph/9905116)

Junkkarinen V., Hewitt A., \& Burbidge G., 1991, Astrophys. J. Suppl. Ser., 77, 203

Kacprzak G. G., Churchill C. W., Steidel C. C., Murphy M. T., \& Evans J. L., 2007, ApJ, 662, 909

Kacprzak G. G., Churchill C. W., Steidel C. C., \& Murphy M. T., 2008, AJ, 135, 922

Kaiser N., \& Peacock J. A., 1991, Astrophys. J., 379, 482

Kayser R., Helbig P., \& Schramm T., 1997, Astron. Astrophys., 318, 680

Kaminker A. D., Ryabinkov A. I., \& Varshalovich D. A., 2000, Astron. Astrophys., 358, 1

Kazin E. A., Blanton M. R., Scoccimarro R. et al., 2010a, Astrophys. J., 710, 1444

Kazin E. A., Blanton M. R., Scoccimarro R., McBridge C. K., Berlind A. A., 2010b, preprint (arXiv:1004.2244)

Kerscher M., Szapudi I., \& Szalay A. S., 2000, Astrophys. J., 535, L13

Kurki-Suonio H., Mathews G. J., \& Fuller G. M., 1990, Astrophys. J., 356, L5
Landy S. D., \& Szalay A. S., 1993, Astrophys. J., 412, 64

Lundgren B. F., Brunner R. J., York D. G. et al., 2009, Astrophys. J., 698, 819

Martínez V. J., Arnalte-Mur P., Saar E., et al., 2009, Astrophys. J., 696, L93;Erratum: 2009, Astrophys. J., 703, L184

Mattig W., 1958, Astron. Nachr., 284, 109

Ménard B., Nestor D., Turnshek D., et al., 2008, Mon. Not. R. Astron. Soc., 385, 1053

Mo H. J., Jing Y. P., \& Börner G., 1992, Astrophys. J., 392, 452

Morikawa M., 1991, Astrophys. J., 369, 20

Peacock J. A., \& Nicholson D., 1991, Mon. Not. R. Astron. Soc., 253, 307

Peebles P. J. E., 1993, Principles of Physical Cosmology, Princeton Univ. Press, Princeton

Percival W. J., Cole S., Eisenstein D. J., et al. 2007a, Mon. Not. R. Astron. Soc., 381, 1053

Percival W. J., Nichol R. C., Eisenstein D. J. et al., 2007b, Astrophys. J., 657, 51

Prochter G. E., Prochaska J. X., \& Burles S. M., 2006, Astrophys. J., 639, 766

Quashnock J. M., Vanden Berk D. E., \& York D. G., 1996, Astrophys. J., 472, L69

Rivolo A. R., 1986, Astrophys. J., 301, 70

Ryabinkov A. I., Kaminker A. D., \& Varshalovich D. A., 2003, Astron. Astrophys., 412, 707; www.ioffe.ru/astro/QC

Ryabinkov A. I., Kaminker A. D., \& Varshalovich D. A., 2007, Mon. Not. R. Astron. Soc., 376, 1838 (Paper I)

Tago E., Saar E., Einasto J. et al., 2002, Astron. J., 123, 37 van de Weygaert R., 1991, Mon. Not. R. Astron. Soc., 249, 159

Yoshida N., Colberg J., White S. D. M. et al., 2001, Mon. Not. R. Astron. Soc., 325, 803

This manuscript was prepared with the AAS LATEX macros v5.2. 


\section{A Toy models for partly ordered SC lattice}

Figs. 8 and 9 represent two models of simulations performed for partly ordered structure of points based on the simple-cubic (SC) lattice and the results of appropriate calculations of power spectra.

Fig. 8 displays a cloud-like distribution of points around the vertices of the SC lattice with unit lattice constant. We consider a superposition of 3 similar lattices with coordinates of the vertices represented as $x_{v}=i_{v}+\delta x_{v}, y_{v}=j_{v}+\delta y_{v}, z_{v}=k_{v}+\delta z_{v}$, where $i_{v}, j_{v}$, and $k_{v}$ are integer numbers within the interval from -50 to +50 with respect to the zero point and $\delta x_{v}, \delta y_{v}$, and $\delta z_{v}$ are random numbers with the Gaussian distribution and standard deviation $\sigma_{v}=0.3$. In other words we have a cloud of three random vertices instead of one lattice vertex.

The radial distribution function for the random points with radial coordinate $r_{v}=\sqrt{x_{v}^{2}+y_{v}^{2}+z_{v}^{2}}$ within a sphere restricted by the radius $R=50$ can be calculated as a value

$\mu=\mu_{\mathrm{R}}\left(r_{l}\right)=\frac{N_{\mathrm{V}}\left(r_{l}\right)-n_{0} 4 \pi r_{l}^{2} \Delta r_{l}}{\sqrt{n_{0} 4 \pi r_{l}^{2} \Delta r_{l}}}$,

where $r_{l}$ is a mean radius of $l$-th concentric layer with fixed radial width (bin) $\Delta r_{l}=0.1, N_{\mathrm{V}}\left(r_{l}\right)$ is a number of vertices within a set of non-overlapping layers, $n_{0}$ is a mean density (number per unit volume) of vertices.

We calculate the power spectra for each realization of $\mu$ given by (A1) using a version of Eq. (7). In this version $r_{\mathrm{m}}$ substitutes for $\delta \eta_{\mathrm{m}}$ and the radius $R$ stands for $\delta \hat{\eta} ; \mu_{\mathrm{m}}=\mu_{\mathrm{R}}\left(r_{\mathrm{m}}\right)$, where the value of $r_{\mathrm{m}}$ runs over a uniform set of points (with a step 0.10) $r_{\mathrm{m}}=$ $0.05,0.15,0.25, \ldots 49.95$ numerated by $m=1,2, \ldots . N_{\mu}$ and $N_{\mu}=500$. By contrast with Eq. (7) we apply here the approach of independent bins (bin=0.1) and put $U \hat{(k)}=1$ in the denominator.

The upper panel in Fig. 8 displays the mean value of the power spectra calculated for 100 realizations of the radial distribution function (A1). The peak with significance $\sim 4.5 \sigma$ (cf. Fig. 4) indicates the presence of oscillations with a period equal to unity (lattice constant).

Similar calculations are represented in Fig. 9 where we use so named hole-like lattices constructed on the base of SC lattice $x_{v}=i_{v}, y_{v}=j_{v}, z_{v}=k_{v}$, where $i_{v}, j_{v}$, and $k_{v}$ are introduced above with a random removing of some vertices. Parameter $u=0.2$ is a ratio of the numbers of residual to initial lattice vertices. Additionally we immerse the hole-like lattice into the field of random Poisson points with sixfold excess of the points over the number of residual vertices $(e=6)$.

Using the version of Eq. (7) discussed above, we calculate the power spectra for different realizations of the combined 3-dimensional distribution of points. The mean value of the power spectra calculated for 100 realizations of the radial distribution function (A1) is represented in the upper panel of Fig. 9. One can see the peak with significance $\sim 4.5 \sigma$ similar to the case of cloud-like lattice.

The lower panels in Figs. 8 and 9 represent 2dimensional illustrations of the partly ordered structures used in the power-spectrum calculations. Let us emphasize a low degree of visual ordering of the structures discussed. 

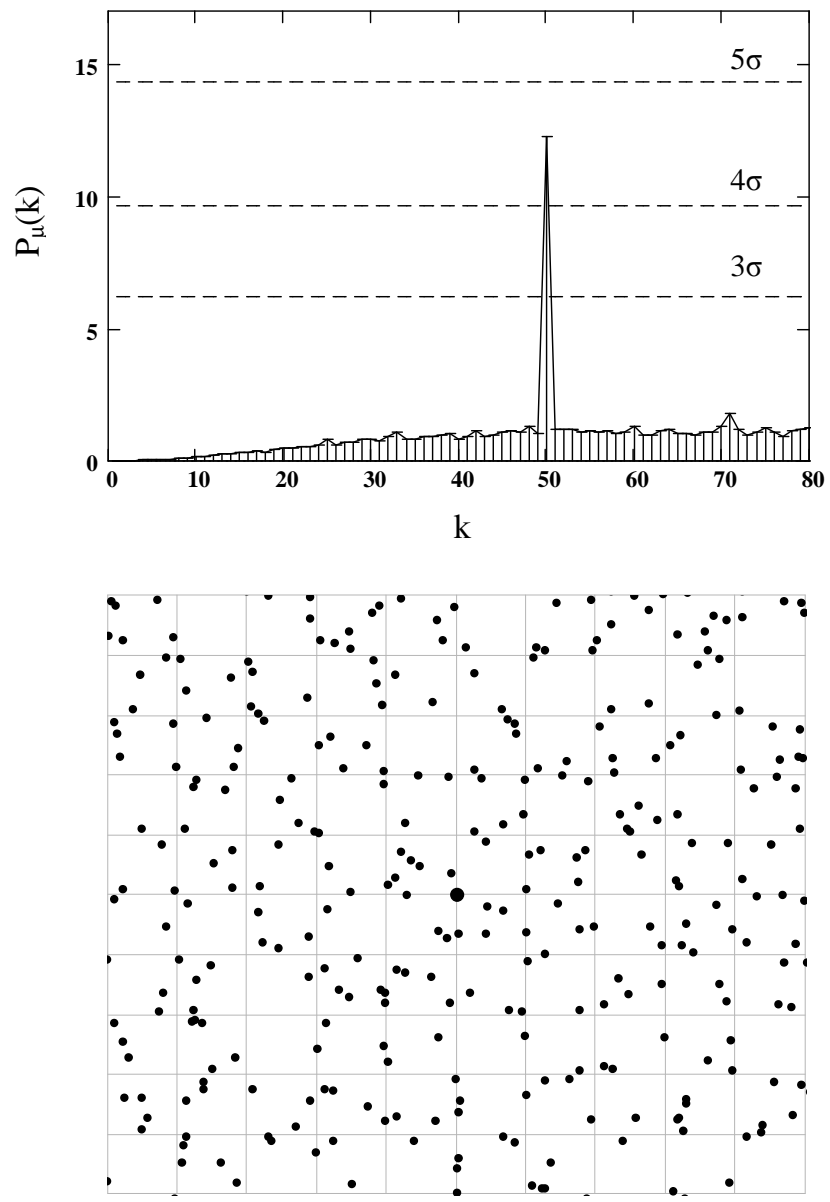

Fig. 8 Upper panel: Mean value of 100 realizations of power spectra calculated for the radial distribution function Eq. A1 in the case of a cloud-like lattice (see text) constructed as superposition of 3 random cubic lattices with the standard deviation $\sigma_{\mathrm{p}}=0.3$ from simple cubic. Lower panel: fragment of 2-dimensional illustration displayed one of 100 realizations of the cloud-like lattice (calculated for the upper panel); points represent lattice vertices and the filled circle indicates the center of the sphere.
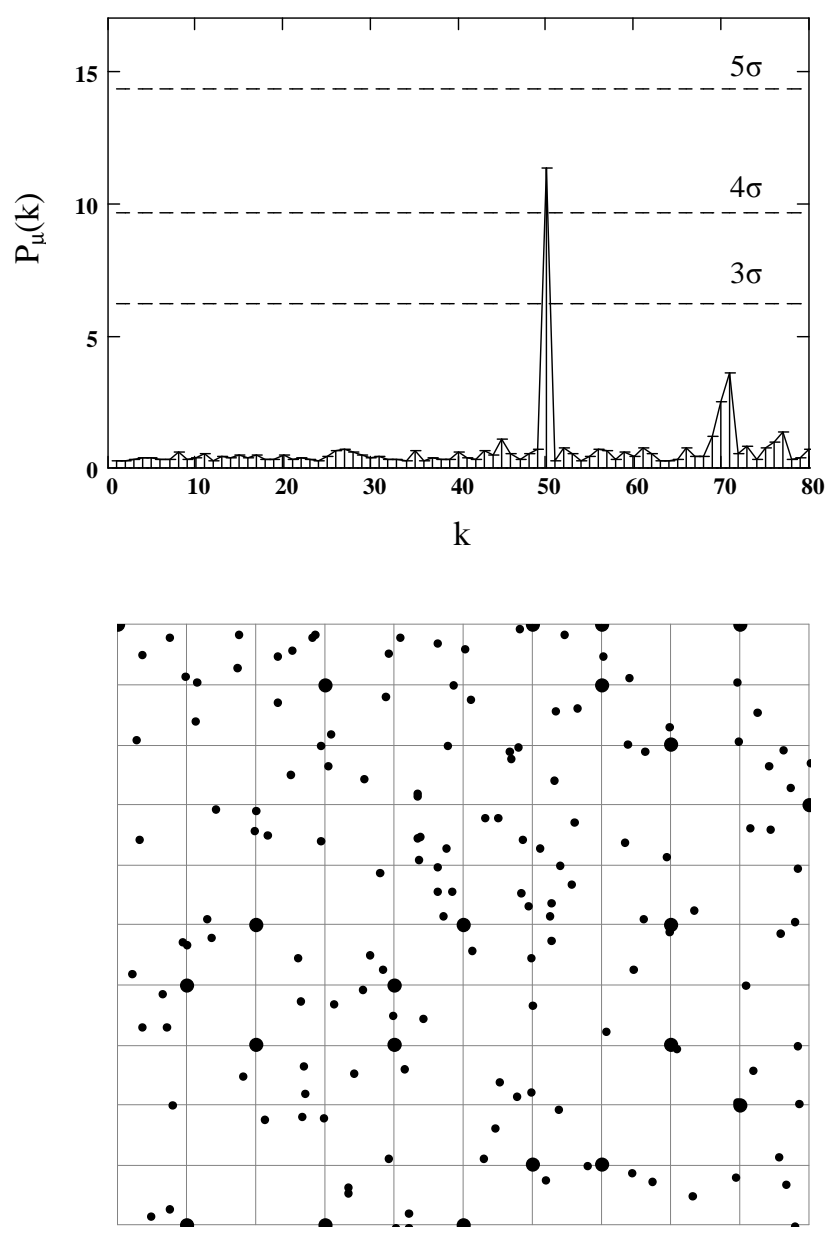

Fig. 9 Same as in Fig. 8 but for superposition of a holelike lattice with a parameter $u=0.2$ (see text) and the field of random Poisson points with sixfold $(e=6)$ excess of random points over hole-like lattice vertices. Upper panel: mean value of 100 realization of power spectra; lower panel: filled circles stand for the vertices of the hole-like lattice. 